\title{
ON THE ALGEBRA OF BOUNDED HOLOMORPHIC MARTINGALES
}

\author{
HITOSHI ARAI
}

\begin{abstract}
The properties of the algebra $H^{\infty}$ of bounded holomorphic martingales are further studied following the work of $N$. Th. Varopoulos [11]. The purpose of this paper is to discuss the behavior of weak* closed algebras $B$ with $H^{\infty} \varsubsetneqq B \varsubsetneqq L^{\infty}$ and the support sets of $H^{\infty}$-functions. We also give some results on the factorization theorem for $H^{\infty}$ and extreme points of the unit ball of $H^{\infty}$.
\end{abstract}

1. Introduction. Let $\left(z_{1}(t): t \geq 0\right), \ldots,\left(z_{m}(t): t \geq 0\right)$ be $m$ independent complex Brownian motions on a complete probability space $(\Omega, P)$ such that

$$
P\left(z_{1}(0)=\cdots=z_{m}(0)=0\right)=1 .
$$

For every $t \geq 0, \mathcal{F}(t)$ denotes the $\sigma$-field generated by $\left\{z_{j}(t): 0 \leq s \leq t ; j=\right.$ $1, \ldots, m\}$ and the $P$-null sets, and $\mathcal{F}$ denotes the $\sigma$-field generated by $\bigcup_{t \geq 0} \mathcal{F}(t)$. Let $S$ be the collection of $(\mathcal{F}(t))$-stopping times. Then for every $T \in S$, we put $\mathcal{F}(T)=\{A \in \mathcal{F}: A \cap\{T \leq t\} \in \mathcal{F}(t)$ for all $t \geq 0\}$.

Let us denote by $H^{\infty}(\Omega)$ the algebra of bounded $(\mathcal{F}(t))$ - martingales $\left(X_{t}: t \geq 0\right)$ which admit an Ito integral representation of the form

$$
X_{t}=X_{0}+\sum_{j=1}^{m} \int_{0}^{t} \alpha_{j}(s) d z_{j}(s) \quad(t \geq 0),
$$

where $\alpha_{1}, \ldots, \alpha_{m}$ are predictable processes. The algebra $H^{\infty}(\Omega)$ was introduced by N. Th. Varopoulos [11], and it was shown that $H^{\infty} \equiv\left\{X_{\infty}:\left(X_{t}: t \geq 0\right) \in H^{\infty}(\Omega)\right\}$ is a weak* Dirichlet algebra on $(\Omega, \mathcal{F}, P)$ (cf. [11, Theorem 3.1]). See [10] for weak* Dirichlet algebras.

Here we investigate properties of $H^{\infty}$. The main purpose of this paper is to prove the following theorem which implies some results as corollaries on the factorization theorem for $H^{\infty}$, extreme points of the unit ball of $H^{\infty}$ and superalgebras of $H^{\infty}$.

THEOREM. (i) Let $T \in S$ with $0<T<\infty$ a.s., and let $A_{T}$ be the weak ${ }^{*}$ closed algebra generated by $H^{\infty}$ and $L^{\infty}(\Omega, \mathcal{F}(T), P)$. Then $H^{\infty} \varsubsetneqq A_{T} \varsubsetneqq L^{\infty}(\Omega, \mathcal{F}, P)$ and $A_{T} \cap \bar{A}_{T}=L^{\infty}(\Omega, \mathcal{F}(T), P)$ (the bar denotes conjugation, here and always). Obviously $H^{\infty}$ is not weak $k^{*}$ maximal.

(ii) For every $A \in \bigcup\{\mathcal{F}(T): T \in S, T<\infty$ a.s. $\}$ and for every two positive numbers $\alpha$ and $\varepsilon$, there exists an $X \in H^{\infty}$ satisfying the following:

(a) $X_{R}=0$ a.s. on the complement of $A$, for an arbitrary $R \in S$,

(b) $0<|X| \leq \alpha$ a.s. on $A$,

(c) $P(A \backslash\{|X|=\alpha\})<\varepsilon$.

Received by the editors May 25, 1984 and, in revised form, May 1, 1985..

1980 Mathematics Subject Classification. Primary 46J15, 60G45.

Key words and phrases. Holomorphic martingales, weak* Dirichlet algebras, Hardy spaces. 
2. Notation. For every subset $D$ of $L^{\infty}(\Omega, \mathcal{F}, P),[D]$ denotes the weak* closed algebra generated by $D$ in $L^{\infty}(\Omega, \mathcal{F}, P)$. For $0<p<\infty$, the closure of $H^{\infty}$ in $L^{p}(\Omega, \mathcal{F}, P)$ will be denoted by $H^{p}$. Suppose that $\mathcal{G}$ is a sub- $\sigma$-field of $\mathcal{F}$. We put $L^{p}(\mathcal{G})=L^{p}(\Omega, \mathcal{G}, P), H^{p}(\mathcal{G})=H^{p} \cap L^{p}(\mathcal{G})$ and $L^{p}=L^{p}(\mathcal{F})(0<p \leq \infty)$.

For $X \in L^{p}$ we write $I(X)$ for the characteristic function of the support set $\operatorname{supp}(X)$ of $X$.

For every Borel set $G \subset \mathbf{C}$, let us denote by $T(G, j)$ the first time at which the Brownian motion $\left(z_{j}(t): t \geq 0\right)$ escapes from $G(1 \leq j \leq m)$. Then we put $D=\bigcup\{F(T(G, j)): G$ is a bounded open subset of $\mathbf{C}$ which contains the origin, and $j=1, \ldots, m\}$.

3. Proof of the Theorem. (1) Since the conditional expectation $E[\cdot \mid \mathcal{F}(T)]$ is multiplicative on $A_{T}$, the proof of Lemma 3 in [8] guarantees that $A_{T} \cap \bar{A}_{T}=$ $L^{\infty}(\mathcal{\digamma}(T))$. Thus (i) is true.

(2) We will construct a bounded holomorphic martingale $X=\left(X_{t}\right)$ with properties (a), (b) and (c) in statement (ii) of the Theorem.

Fix an $\left(\mathcal{F}_{t}\right)$-stopping time $T$ such that $A \in \mathcal{F}(T)$ and $T<\infty$ a.s. Let $T(n)=$ $\inf \left\{t:\left|z_{1}(t)\right|>n\right\}$ and $X_{t}^{(n)}=z_{1}(T(n) \wedge t), t \geq 0 ; n \in \mathbf{N}$. Define a holomorphic martingale $Y^{(n)}=\left(Y_{t}^{(n)}\right)$ by $Y^{(n)}=\int a d X^{(n)}$, where

$$
a(s, \omega)=\left\{\begin{array}{ll}
0 & (0 \leq s \leq T(\omega)), \quad(\omega \in \Omega) . \\
1 & (T(\omega)<s)
\end{array} \quad .\right.
$$
let

Write $A_{1}=A \cap\{T<T(1)\}$ and $A_{n}=A \cap\{T(n-1) \leq T<T(n)\}(n \geq 2)$, and

$$
R(n)= \begin{cases}T & \text { on }\left(A_{n}\right)^{c}, \text { where }\left(A_{n}\right)^{c} \text { is the complement of } A_{n}, \\ \infty & \text { on } A_{n}\end{cases}
$$

( $n \in \mathbf{N}$ ). Since $A_{n}$ belongs to $\mathcal{F}(T), R(n)$ is an $\left(\xi_{t}\right)$-stopping time. Obviously we have that $\{R(n)>T\}=\{R(n)=\infty\}=A_{n} \subset\{T(n)>T\}$. This yields that

$$
\begin{aligned}
P(R(n)>T) & =P\left(\left\{z_{1}(T(n)) \neq z_{1}(T)\right\} \cap\{R(n)>T\}\right) \\
& =P\left(\left\{z_{1}(T(n) \wedge R(n)) \neq z_{1}(T(n) \wedge T)\right\} \cap\{R(n)>T\}\right) \\
& =P\left(\left\{X_{R(n)}^{(n)} \neq X_{T}^{(n)}\right\} \cap\{R(n)>T\}\right) .
\end{aligned}
$$

Hence the support set of $Y_{R(n)}^{(n)}$ is $A_{n}(n \in \mathbf{N})$, that is, $Y_{R(n)}^{(n)}=0$ a.s. on $\left(A_{n}\right)^{c}$ and $Y_{R(n)}^{(n)} \neq 0$ a.s. on $A_{n}$. By this fact there exists a $\left.\delta_{n} \in\right] 0,1[$ with

$$
P\left(A_{n} \backslash\left\{\left|Y_{R(n)}^{(n)}\right|>\delta_{n}\right\}\right)<\left(\varepsilon / 2^{n}\right)
$$

Put $S(n)=\inf \left\{t:\left|Y_{R(n) \wedge t}^{(n)}\right| \geq \delta_{n}\right\}$. It is easy to check that the support set of $Y_{R(n) \wedge S(n)}^{(n)}$ is $A_{n}(n \in \mathrm{N})$. Since $A_{1}, A_{2}, \ldots$ are mutually disjoint, we get that $\lim _{k \rightarrow \infty} \sum_{n=1}^{k}\left(\alpha / 2^{n} \delta_{n}\right) Y_{R(n) \wedge S(n)}^{(n)}$ exists a.s. and $\left|\sum_{n=1}^{\infty}\left(\alpha / 2^{n} \delta_{n}\right) Y_{R(n) \wedge S(n)}^{(n)}\right| \leq \alpha$ a.s. Hence by the Krein-Smulian consequence (cf. [2, Lemma 3.5]), we obtain that

$$
X \equiv \sum_{n=1}^{\infty}\left(\alpha / 2^{n} \delta_{n}\right) Y_{R(n) \wedge S(n)}^{(n)}
$$


belongs to $H^{\infty}$. Further $A \supset \bigcup_{n=1}^{\infty} A_{n}$ and $P\left(A \backslash\left(\bigcup_{n-1}^{\infty} A_{n}\right)\right)=0$, because $\max \left\{\left|z_{1}(t)(\omega)\right|: 0 \leq t \leq T(\omega)\right\}<\infty$ a.s. $\omega \in \Omega$. From this and the definition of $X$ it follows that $X$ satisfies desired properties.

4. Applications of the Theorem. Recently T. Nakazi introduced the following canonical algebras $H_{\min }^{\infty}$ and $H_{\max }^{\infty}$ which play an important role in the theory of abstract Hardy algebras [7]:

$$
\begin{aligned}
& H_{\min }^{\infty}=\bigcap\left\{B: B \text { is a weak }{ }^{*} \text { closed algebra with } H^{\infty} \varsubsetneqq B \subset L^{\infty}\right\}, \\
& H_{\max }^{\infty}=\left[H^{\infty}, I(X): X \in H^{\infty}\right] .
\end{aligned}
$$

As an application of the Theorem we first describe the canonical algebras in the case of our algebras $H^{\infty}$.

Corollary 1. (1) $H_{\min }^{\infty}=H^{\infty}$. (2) $H_{\max }^{\infty}=L^{\infty}$.

Proof. By part (i) of the Theorem and [4, p. 79, Lemma 6.1] we have $\left(\bigcap_{t>0} A_{t}\right) \cap\left(\bigcap_{t>0} A_{t}\right)^{-}=\bigcap_{t>0}\left(A_{t} \cap \bar{A}_{t}\right)=\bigcap_{t>0} L^{\infty}(\mathcal{F}(t))=L^{\infty}(\mathcal{F}(0))=\mathbf{C}$. Hence, by $\left[6\right.$, pp. 516-517] we obtain $\bigcap_{t>0} A_{t}=H^{\infty}$, proving Corollary 1(1). Corollary 1(2) is an immediate consequence of part (ii) of the Theorem.

REMARK. Compare the above description of the canonical algebras with the following classical result: Let $H^{\infty}(\Gamma)$ be the classical Hardy algebra on the unit circle $\Gamma$. Then $H_{\text {min }}^{\infty}(\Gamma)=L^{\infty}(\Gamma)$ and $H_{\text {max }}^{\infty}(\Gamma)=H^{\infty}(\Gamma)$ (see Hoffman $[3$, pp. 194, 52]).

We next consider the factorization theorem of Srinivasan-Wang type for $H^{\infty}$.

Corollary 2. Suppose $0<p<\infty$. Then for every $X \in L^{p}$ satisfying $\operatorname{supp}(X) \in D$ and $E[I(X) \log |X|]>-\infty$, there exist $q, g$ and $h$ such that (1) $f=q|g| h$, (2) $|q|=1$ a.s. and $q \in\left[X H^{\infty}\right]_{p}$, (3) $h \in H^{p}$ and is outer, and (4) $g \in H^{\infty}$ and $|g|=I(X)$ a.s., where $\left[X H^{\infty}\right]_{p}$ is the $L^{p}$-closure of $X H^{\infty}$.

If $1 \leq p<\infty$ and $E[\log |X|]>-\infty$, then Corollary 2 implies the usual factorization of $X$, because $I(X)=1$ a.s and $\operatorname{supp}(X)=\Omega \in D$.

Let $d(\cdot, \cdot)$ be a metric introduced by Gamelin and Lumer, and let $H$ be the universal Hardy class over $H^{\infty}$ (cf. [2, p. 122] ). For every $u \in \operatorname{Re}\left(L^{1}\right)$ we denote by ${ }^{*} u$ the conjugate function of $u$ (cf. $[2$, pp. 124, 147]).

Proof of Corollary 2. Let $f=X+1-I(X)$. Define $F_{n}$ as follows:

$$
F_{n}= \begin{cases}|f| & \text { on }\{(1 / n) \leq|f| \leq n\} \\ 1 / n & \text { on }\{|f|<(1 / n)\} \\ 1 & \text { on }\{n<|f|\}\end{cases}
$$

Then it is easy to check that $\lim _{n \rightarrow \infty}\left\|\log \left(F_{n}\right)-\log |f|\right\|_{1}=0$. We put $G_{n}=$ $\exp \left[\log \left(F_{n}\right)+i^{*}\left(\log \left(F_{n}\right)\right)\right]$ and $K_{n}=\exp \left[-\log \left(F_{n}\right)-i^{*}\left(\log \left(F_{n}\right)\right)\right]$. Then $G_{n}, K_{n} \in$ $H^{\infty}(n \in \mathbf{N})$. By Kolmogorov's inequality (cf. [12]), there exists a real valued $\mathcal{F}$-measurable function $w$ such that the sequence $\left\{{ }^{*}\left(\log \left(F_{n}\right)\right)\right\}$ converges to $w$ in measure. Since $\lim _{n \rightarrow \infty} F_{n}=f$ a.s., there is a subsequence $\{n(k)\}$ of $\{n\}$ such that $\lim _{k \rightarrow \infty}\left[\log \left(F_{n(k)}\right)+i^{*}\left(\log \left(F_{n(k)}\right)\right)\right]=\log |f|+i w$ a.s. Let $h=\exp [\log |f|+i w]$ and let $K=\exp [-\log |f|-i w]$. It is not hard to see that $\lim _{k \rightarrow \infty}\left\|G_{n(k)}-h\right\|_{p}=0$ and $\lim _{k \rightarrow \infty} d\left(K_{n(k)}, K\right)=0$. Hence $h \in H^{p}, K \in H$ and $h K=1$. So $h$ is outer. Let $q=K f$. Since we have easily that $|q|=1$ a.s. and $\lim _{k \rightarrow \infty}\left\|K_{n(k)} f-q\right\|_{p}=0$, 
$q \in\left[X H^{\infty}\right]_{p}$. By an easy modification of the proof of part (ii) of the Theorem we obtain that there exists $g \in H^{\infty}$ such that $|g|=I(X)$ a.s. Thus $q, h$ and $g$ are the desired factorization.

From the Theorem we also obtain the following

COROLlary 3. Let $T \in S$ with $T<\infty$ a.s. Let $X \in H^{\infty}$. Then $X_{T}$ is an extreme point of the unit ball of $H^{\infty}$ if and only if $\left|X_{T}\right|=1$ a.s.

We omit the proof.

5. Weak* closed algebras which contain $H^{\infty}$. Let $\tau=\inf \left\{t:\left|z_{1}(t)\right| \geq 1\right\}$. Then Corollary 1(1) yields the following

COROLLARY 4. Let $B$ be the complete $\sigma$-field generated by $z_{1}(\tau)$. Let $B$ be the weak ${ }^{*}$ closed algebra between $H^{\infty}$ and $L^{\infty}$. Then the following are equivalent.

(1) $B=H^{\infty}$.

(2) $B \cap \bar{B} \subset L^{\infty}(B)$.

ProOF. Since the proof of "(1) $\rightarrow(2)$ " is obvious, we need prove only " $(2) \rightarrow$ (1)". By (2) and [5, Proposition 1.4], we have $B \subset\left[H^{\infty}, z_{1}(\tau)^{-}\right]$. If $H^{\infty} \underset{\ddagger}{\subsetneq} B$, then there exists a weak* closed algebra $D$ such that $H^{\infty} \varsubsetneqq D \varsubsetneqq B \subset\left[H^{\infty}, z_{1}(\tau)^{-}\right]$by Corollary 1(1) [9, Corollary 1 and 5, p. 54]. It is easy to check that $H^{\infty}(B)$ is a maximal weak* Dirichlet algebra on $(\Omega, B, P)$. Hence $D \cap L^{\infty}(B)=H^{\infty}(B)$. Since $D \cap \bar{D} \subset B \cap \bar{B} \subset L^{\infty}(B)$, we have

$$
D \cap \bar{D}=\left(D \cap L^{\infty}(B)\right) \cap\left(D \cap L^{\infty}(B)\right)^{-}=H^{\infty}(B) \cap H^{\infty}(B)^{-}=\mathbf{C} .
$$

By $\left[6\right.$, pp. 516-517] we obtain $D=H^{\infty}$. But this contradicts the definition of $D$. Thus $B=H^{\infty}$.

COROLlaRY 5. Let $\mathcal{G}$ be a sub- $\sigma$-field of $B$. Then the following are equivalent.

(1) $E[\cdot \mid \mathcal{G}]$ is multiplicative on $H^{\infty}$.

(2) $\mathcal{G}$ is a trivial $\sigma$-field.

Hence an operator $N(X)\left(e^{i \theta}\right)=E\left[X \mid z_{1}(\tau)=e^{i \theta}\right]$ is not multiplicative on $H^{\infty}$.

REMARK. The operator $N$ was introduced by N. Th. Varopoulos (cf. [11]). K. Carne [1] pointed out that $N$ is not multiplicative on $H^{\infty}$. He showed more generally that there is no algebra homomorphism from $H^{\infty}$ to $H^{\infty}(\Gamma)$. However, Corollary 5 is not a special case of Carne's result, because we do not assume $E\left[H^{\infty} \mid \mathcal{g}\right] \subset H^{\infty}$.

PROOF OF COROLlaRY 5. Since $(2) \rightarrow(1)$ is a well-known result (cf. [11]), it is sufficient to show the converse $(1) \rightarrow(2)$.

Suppose that $E[\cdot \mid \mathcal{G}]$ is multiplicative on $H^{\infty}$. Let $B=\left[H^{\infty}, L^{\infty}(\mathcal{G})\right]$. By the same proof as in Lemma 3 in [8] we have $B \cap \bar{B}=L^{\infty}(\mathcal{G}) \subset L^{\infty}(B)$. Hence $B=H^{\infty}$ by Corollary 4 . Thus $\mathcal{G}$ is the trivial $\sigma$-field.

\section{REFERENCES}

1. K. Carne, The algebra of bounded holomorphic martingales, J. Funct. Anal. 45 (1982), 95-108.

2. T. W. Gamelin and G. Lumer, Theory of abstract Hardy spaces and the universal Hardy class, Adv. in Math. 2 (1968), 118-174. 
3. K. Hoffman, Banach spaces of analytic functions, Prentice-Hall, Englewood Cliffs, N.J., 1962.

4. N. Ikeda and S. Watanabe, Stochastic differential equations and diffusion processes, North-Holland, Amsterdam, and Kodansha, Tokyo, 1981.

5. R. Kallenborn and H. König, An invariant subspace theorem in the abstract Hardy algebra theory, Arch. Math. 39 (1982), 51-58.

6. P. S. Muhly, Maximal weak* Dirichlet algebras, Proc. Amer. Math. Soc. 36 (1972), 515518.

7. T. Nakazi, Superalgebras of weak* Dirichlet algebras, Pacific J. Math. 68 (1977), 197-207.

8. __ Extended weak* Dirichlet algebras, Pacific J. Math. 81 (1979), 493-513.

9. __ Minimal superalgebras of weak* Dirichlet algebras, preprint.

10. T. P. Srinivasan and Ju-Kwei Wang, Weak* Dirichlet algebras, Proc. Internat. Sympos. on Function Algebras (Tulane Univ., 1965), Scott Foresman, Glenview, Ill., 1966, pp. 216-249.

11. N. Th. Varopoulos, The Helson-Szegö theorem and $A_{p}$-functions for Brownian motion and several variables, J. Funct. Anal. 39 (1980), 85-121.

12. K. Yabuta, Kolmogorov's inequalities in the abstract Hardy space theory, Arch. Math. 30 (1978), 418-421.

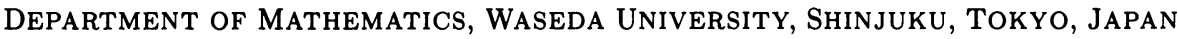

